\title{
Measures on Reducing the Recurrence of Colorectal Polyps
}

\author{
Fan Chen* \\ Department of Gastroenterology, Fuzhou General Hospital, Fuzhou, China \\ *Corresponding author: Fan Chen, Fuzhou General Hospital, Dongfangyuan, 156 North West Second Ring Road, Fuzhou City, China \\ To Cite This Article: Fan Chen, Measures on Reducing the Recurrence of Colorectal Polyps. 2020 - 7(6). AJBSR.MS.ID.001204. \\ DOI: 10.34297/AJBSR.2020.07.001204.
}

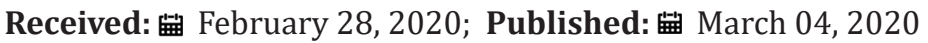

\section{Opinion}

A large number previous studies have confirmed that, the generation of colorectal cancer follows the sequential pattern from adenoma to colorectal cancer, so colorectal polyps, especially adenomatous colorectal polyps, are regarded as precancerous lesions of colorectal cancer, and the timely detection and removal of colorectal polyps has become an important means to prevent the occurrence of colorectal cancer. However, many studies at home and abroad suggest that there is a high recurrence rate after colorectal polypectomy, which brings inconvenience to patients' lives and health risks, Therefore, in addition to regular endoscopic follow-up, We had better cooperate with some anti recurrence measures, as described below.

Firstly, patients should adjust their living and eating habits related to diseases. The risk factors of colorectal polyps are tobacco, alcohol, high fat and low fiber diet, abdominal obesity, mental stimulation, etc., while calcium, aspirin, trace elements iron and zinc, as well as the intake of multi vitamins are the protective factors of colorectal polyps. Therefore, in life, we should stop smoking, avoid excessive drinking, eat less greasy food, eat less meat food, control the level of body lipids, maintain normal weight, avoid bad mental stimulation, eat less cold and raw food, also should eat more vegetables, fruits and fiber food, and exercise properly.

Secondly, during the endoscopic treatment period, colorectal polyp should be removed as clean as possible. For the smaller polyp, we can remove them by forceps. For larger polyps, because of increased risk of recurrence, we had better remove the polyps clean under the condition of ensuring the safety of patients, so as to make sure that there are no residual adenoma cells on the edge of resection in pathology. Some studies have shown that the recurrence rate of colorectal polyps with endoscopic mucosal dissection in similar patients is lower than that with endoscopic mucosal resection. In addition, we should be serious and careful to minimize missed diagnosis when searching for lesions by endoscopy.

Lastly, appropriate drug intervention might be performed for risk patients after polypectomy. For patients with colorectal polyps, the risk of recurrence should be assessed after polypectomy, such as gender, age, nature of colorectal polyps number, size, growth location, pathological type), etc., and for those with higher risk of recurrence, such as men, family history, age $\geq 50$ years old, number of intestinal polyps $\geq 3$, diameter of polyps $\geq 11 \mathrm{~mm}$, and the pathological type being adenomatous polyps, in addition to shortening the time interval of endoscopic follow-up properly, drug therapy should also be carried out. In western medicine, probiotics and calcium are recommended, and in traditional Chinese medicine, Shenling Baizhu powder and Wumei Pill are recommended. Studies have shown that oral probiotics can correct the imbalance of intestinal microecology, relieve the symptoms of diarrhea and constipation, inhibit intestinal inflammation, and prevent recurrence and canceration of colorectal adenomas. Because the patients with colorectal polyps are mostly middleaged and elderly people, more or less accompanied with different degrees of osteoporosis, we can use calcium combined with vitamin D as appropriate for such patients, but in the process of taking, we should prevent gastrointestinal discomfort, and monitor the blood calcium concentration of patients. In addition, according to the dialectical situation of patients, we should use Shenling Baizhu powder and Wumei Pill as appropriate.

In summary, since colorectal polyps are easy to recur, we should pay attention to them, be careful in diagnosis and treatment. After 
treatment, we should adjust personal living habits and eating habits, accordingly, strictly follow up regularly, and take relevant drugs as appropriate, if so, we can reduce the recurrence of polyps, reduce the possibility of canceration, and ultimately benefit patients. 